\title{
Propagation of mutual intensity expressed in terms of the fractional Fourier transform
}

\author{
M. Fatih Erden and Haldun M. Ozaktas \\ Department of Electrical Engineering, Bilkent University, 06533 Bilkent, Ankara, Turkey \\ David Mendlovic \\ Faculty of Engineering, Tel Aviv University, 69978 Tel Aviv, Israel
}

Received April 24, 1995; revised manuscript received July 28, 1995; accepted September 29, 1995

\begin{abstract}
The propagation of mutual intensity through quadratic graded-index media or free space can be expressed in terms of two-dimensional fractional Fourier transforms for one-dimensional systems and in terms of fourdimensional fractional Fourier transforms for two-dimensional systems. As light propagates, its mutual intensity distribution is continually fractional Fourier transformed. These results can also be generalized to arbitrary first-order optical systems. Furthermore, the Wigner distribution associated with a partially coherent field rotates in the same manner as the Wigner distribution associated with a deterministic field.

Key words: diffraction, Fourier optics, statistical optics, fractional Fourier transforms, mutual intensity.

(C) 1996 Optical Society of America
\end{abstract}

\section{INTRODUCTION}

The $a$ th order fractional Fourier transform $\left(\mathcal{F}^{a} \hat{q}\right)(u)$ of the function $\hat{q}(u)$ is defined for $0<|a|<2$ as

$$
\begin{aligned}
\left(\mathcal{F}^{a} \hat{q}\right)(u) \equiv & \int_{-\infty}^{\infty} B_{a}\left(u, u^{\prime}\right) \hat{q}\left(u^{\prime}\right) \mathrm{d} u^{\prime}, \\
B_{a}\left(u, u^{\prime}\right) \equiv & \frac{\exp [-i(\pi \hat{\phi} / 4-\phi / 2)]}{|\sin \phi|^{1 / 2}} \exp \left[i \pi \left(u^{2} \cot \phi\right.\right. \\
& \left.\left.-2 u u^{\prime} \csc \phi+u^{\prime 2} \cot \phi\right)\right],
\end{aligned}
$$

where

$$
\phi \equiv a \pi / 2
$$

and $\hat{\phi}=\operatorname{sgn}(\sin \phi)$. The kernel is defined separately for $a=0$ and $a= \pm 2$ as $B_{0}\left(u, u^{\prime}\right) \equiv \delta\left(u-u^{\prime}\right)$ and $B_{ \pm 2}\left(u, u^{\prime}\right) \equiv$ $\delta\left(u+u^{\prime}\right)$, respectively. ${ }^{1}$ The definition is easily extended outside the interval $[-2,2]$ by noting that $\mathcal{F}^{4 j+a} \hat{q}=\mathcal{F}^{a} \hat{q}$ for any integer $j$. Both $u$ and $u^{\prime}$ are interpreted as dimensionless variables.

Some essential properties of the fractional Fourier transform are (1) it is linear; (2) the first-order transform $(a=1)$ corresponds to the common Fourier transform; (3) it is additive in index, $\mathcal{F}^{a_{1}} \mathcal{F}^{a_{2}} \hat{q}=\mathcal{F}^{a_{1}+a_{2}} \hat{q}$; (4) the kernel for the -ath-order transform is the conjugate of the kernel for the ath-order transform: $B_{a}^{*}\left(u, u^{\prime}\right)=B_{-a}\left(u, u^{\prime}\right)$. Other properties may be found in Refs. 1-9.

Optical implementations of the fractional Fourier transform have already been presented. In Refs. 2-4 the fractional-Fourier-transforming property of quadratic graded-index media is discussed. In Refs. 6 and 10 bulk optical systems are considered. Signal-processing applications have been suggested in these references and in Refs. 5, 7, and 9. Further development of the role of the fractional Fourier transform in optics, as well as certain extensions and experimental results, may be found in Refs. $2-4$ and $11-15$

In Refs. 10 and 15 it is shown that there exists a fractional-Fourier-transform relation between the amplitude distributions of light on two spherical surfaces of given radii and separation. Unlike most other papers that deal with the implementation of the fractional transform, these papers pose the transform as a tool for analyzing and describing optical systems composed of an arbitrary sequence of thin lenses and sections of free space. The fractional transform allows one to express the evolution of the amplitude distribution of light through an optical system in terms of fractional Fourier transforms of increasing order. The present paper extends these results to partially coherent light by formulating the propagation of mutual intensity in terms of the fractional Fourier transform.

In all of the references mentioned above, statistical properties of light are ignored and full coherence is assumed. In some cases, however, this assumption cannot be justified, and so the wave functions must be considered as random processes. One of the important quantities used to describe the statistical properties of light is its mutual intensity. Assuming quasi-monochromatic light, the mutual intensity can be expressed as ${ }^{16,17}$,

$$
J\left(r_{1}, r_{2}\right)=E\left[U\left(r_{1}\right) U^{*}\left(r_{2}\right)\right],
$$

where $E[\cdot]$ is the expected value operator and $U(r)$ is the complex amplitude distribution of the optical wave.

For simplicity, we restrict our attention to onedimensional systems. The extension to two-dimensions is straightforward.

This is not the only application of fractional Fourier transforms to optical systems with partially coherent light. In Ref. 18 the output intensity of such systems 
is related to the fractional Fourier transform of the input, where the order $a$ is related to the degree of partial coherence.

\section{PROPAGATION OF MUTUAL INTENSITY THROUGH OPTICAL SYSTEMS}

Quasi-monochromatic light propagates through a linear medium according to the equation

$$
U_{\text {out }}(x)=\int_{-\infty}^{\infty} h\left(x, x^{\prime}\right) U_{\text {in }}\left(x^{\prime}\right) \mathrm{d} x^{\prime},
$$

where $U_{\text {in }}\left(x^{\prime}\right)$ and $U_{\text {out }}(x)$ are the input and the output complex amplitude distributions of the optical waves, respectively, and $h\left(x, x^{\prime}\right)$ is the kernel characterizing the medium. If we use the definition of mutual intensity given in Eq. (3), the output mutual intensity $J_{\text {out }}\left(x_{1}, x_{2}\right)$ can be related to the input mutual intensity $J_{\text {in }}\left(x_{1}{ }^{\prime}, x_{2}{ }^{\prime}\right)=$ $E\left[U_{\text {in }}\left(x_{1}{ }^{\prime}\right) U_{\text {in }}^{*}\left(x_{2}{ }^{\prime}\right)\right]$ as

$$
\begin{aligned}
J_{\text {out }}\left(x_{1}, x_{2}\right)= & E\left[U_{\text {out }}\left(x_{1}\right) U_{\text {out }}^{*}\left(x_{2}\right)\right], \\
J_{\text {out }}\left(x_{1}, x_{2}\right)= & E\left[\int_{-\infty}^{\infty} \int_{-\infty}^{\infty} h\left(x_{1}, x_{1}{ }^{\prime}\right) h^{*}\left(x_{2}, x_{2}{ }^{\prime}\right)\right. \\
& \left.\times U_{\text {in }}\left(x_{1}^{\prime}\right) U_{\text {in }}^{*}\left(x_{2}{ }^{\prime}\right) \mathrm{d} x_{1}{ }^{\prime} \mathrm{d} x_{2}{ }^{\prime}\right], \\
J_{\text {out }}\left(x_{1}, x_{2}\right)= & \int_{-\infty}^{\infty} \int_{-\infty}^{\infty} h\left(x_{1}, x_{1}{ }^{\prime}\right) h^{*}\left(x_{2}, x_{2}{ }^{\prime}\right) \\
& \times J_{\text {in }}\left(x_{1}{ }^{\prime}, x_{2}{ }^{\prime}\right) \mathrm{d} x_{1}{ }^{\prime} \mathrm{d} x_{2}{ }^{\prime} .
\end{aligned}
$$

In these equations $x_{1}, x_{2}$, etc., have dimensions of length.

\section{A. Quadratic Graded-Index Media}

Let us look at the propagation of light through a quadratic graded-index (GRIN) medium. The refractive-index distribution of quadratic GRIN media is given by ${ }^{10}$

$$
n^{2}(x)=n_{0}^{2}\left[1-(x / \xi)^{2}\right]
$$

where $n_{0}>0$ and $\xi>0$ are the medium parameters. In this equation, $n_{0}$ is the refractive index along the optical axis and $\xi$ has dimensions of length. It is shown in Refs. 5 and 10 that a piece of quadratic GRIN medium of length $a \xi \pi / 2$ acts as an $a$ th-order fractional Fourier transformer. More precisely, the kernel characterizing the quadratic GRIN medium, $h\left(x, x^{\prime}\right)$, is related to the fractional-Fourier-transform kernel $B_{a}\left(x, x^{\prime}\right)$ through the relation

$$
h\left(x, x^{\prime}\right)=\exp \left[i\left(k n_{0} a L-a \pi / 4\right)\right] s^{-1} B_{a}\left(x / s, x^{\prime} / s\right),
$$

where $k=2 \pi / \lambda, L$ is the length of the medium, and $s=\sqrt{\lambda \xi / n_{0}}$ ( $\lambda$ is the wavelength). $s$ has units of length. If we substitute this kernel expression into Eq. (5), the output mutual intensity can be related to the input mutual intensity by

$$
\begin{aligned}
J_{\text {out }}\left(x_{1}, x_{2}\right)= & \int_{-\infty}^{\infty} \int_{-\infty}^{\infty} \frac{1}{s^{2}} B_{a}\left(\frac{x_{1}}{s}, \frac{x_{1}^{\prime}}{s}\right) B_{a}^{*}\left(\frac{x_{2}}{s}, \frac{x_{2}^{\prime}}{s}\right) \\
& \times J_{\text {in }}\left(x_{1}^{\prime}, x_{2}^{\prime}\right) \mathrm{d} x_{1}^{\prime} \mathrm{d} x_{2}^{\prime}
\end{aligned}
$$

or

$$
\begin{aligned}
J_{\text {out }}\left(x_{1}, x_{2}\right)= & \int_{-\infty}^{\infty} \int_{-\infty}^{\infty} \frac{1}{s^{2}} B_{a}\left(\frac{x_{1}}{s}, \frac{x_{1}{ }^{\prime}}{s}\right) B_{-a}\left(\frac{x_{2}}{s}, \frac{x_{2}{ }^{\prime}}{s}\right) \\
& \times J_{\text {in }}\left(x_{1}{ }^{\prime}, x_{2}{ }^{\prime}\right) \mathrm{d} x_{1}{ }^{\prime} \mathrm{d} x_{2}{ }^{\prime} .
\end{aligned}
$$

When we look at Eq. (9) we see that the output mutual intensity is essentially the two-dimensional fractional Fourier transform of the input mutual intensity, apart from the sign reversal of the order along one of the dimensions.

\section{B. Propagation through Free Space}

It is shown in Refs. 10 and 15 that there exists a fractional-Fourier-transform relation between the amplitude distributions of light on two spherical surfaces of given radii and separation (Fig. 1). In other words, the amplitude distribution of light on the second surface can be expressed as the fractional Fourier transform of that on the first surface (provided that the radii and separation of the surfaces satisfy a certain inequality). Referring to Fig. 1, the complex amplitude distributions with respect to the first and second spherical reference surfaces are denoted by $U_{\text {in }}\left(x^{\prime}\right)$ and $U_{\text {out }}(x)$, respectively. $\quad R_{1}$ and $R_{2}$ are defined as the radii of the spherical surfaces, and $d$ is the distance between the planar surfaces.

From Ref. 10, the kernel describing propagation between the two surfaces can be expressed as

$$
\begin{aligned}
h\left(x, x^{\prime}\right)= & \frac{\exp (i 2 \pi d / \lambda) \exp [i(\pi \hat{\phi} / 4-\phi / 2)]|\sin \phi|^{1 / 2}}{\sqrt{i \lambda d}} \\
& \times B_{a}\left(\frac{x}{s_{2}}, \frac{x^{\prime}}{s_{1}}\right)
\end{aligned}
$$

with

$$
\begin{aligned}
s_{1} & =(\lambda d)^{2}\left(g_{1} / g_{2}-g_{1}{ }^{2}\right)^{-1 / 4}, \\
s_{2} & =(\lambda d)^{2}\left(g_{2} / g_{1}-g_{2}{ }^{2}\right)^{-1 / 4}, \\
\tan (\phi) & = \pm\left[\left(1 / g_{1} g_{2}\right)-1\right]^{1 / 2},
\end{aligned}
$$

where $\phi=a \pi / 2, g_{1}=1+d / R_{1}, g_{2}=1-d / R_{2}$, and $\lambda$ is the wavelength. In Eq. (13), \pm is determined according to the common sign of $g_{1}$ and $g_{2}$, and $\phi$ is assumed to lie in the interval $[0, \pi]$. All the equations from Eq. (10) to Eq. (13) are valid only if $0 \leq g_{1} g_{2} \leq 1$. Otherwise, the

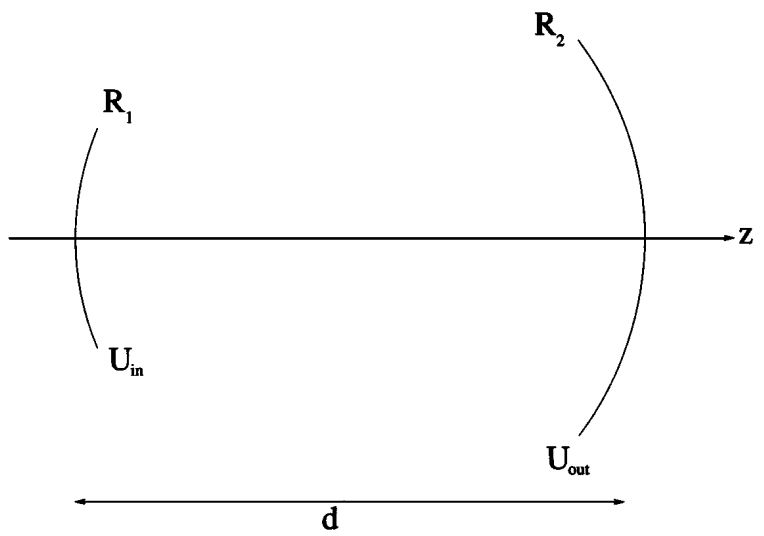

Fig. 1. Here the figure is drawn such that $R_{1}<0$ and $R_{2}>0$. The distance $d$ is always taken to be positive. 
kernel describing propagation between the two spherical surfaces cannot be expressed in terms of the fractionalFourier-transform kernel with a real fractional-order parameter $a$.

If we substitute the kernel expression given in Eq. (10) into Eq. (5), the output mutual intensity can be related to the input mutual intensity by

$$
\begin{aligned}
J_{\text {out }}\left(x_{1}, x_{2}\right)= & \frac{|\sin (\phi)|}{\lambda d} \int_{-\infty}^{\infty} \int_{-\infty}^{\infty} B_{a}\left(\frac{x_{1}}{s_{2}}, \frac{x_{1}{ }^{\prime}}{s_{1}}\right) \\
& \times B_{a}^{*}\left(\frac{x_{2}}{s_{2}}, \frac{x_{2}{ }^{\prime}}{s_{1}}\right) J_{\text {in }}\left(x_{1}{ }^{\prime}, x_{2}{ }^{\prime}\right) \mathrm{d} x_{1}{ }^{\prime} \mathrm{d} x_{2}{ }^{\prime}
\end{aligned}
$$

or

$$
\begin{aligned}
J_{\text {out }}\left(x_{1}, x_{2}\right)= & \frac{|\sin (\phi)|}{\lambda d} \int_{-\infty}^{\infty} \int_{-\infty}^{\infty} B_{a}\left(\frac{x_{1}}{s_{2}}, \frac{x_{1}{ }^{\prime}}{s_{1}}\right) \\
& \times B_{-a}\left(\frac{x_{2}}{s_{2}}, \frac{x_{2}{ }^{\prime}}{s_{1}}\right) J_{\text {in }}\left(x_{1}{ }^{\prime}, x_{2}{ }^{\prime}\right) \mathrm{d} x_{1}{ }^{\prime} \mathrm{d} x_{2}{ }^{\prime} .
\end{aligned}
$$

Just as in the quadratic GRIN case, we see that the output mutual intensity is essentially the two-dimensional fractional Fourier transform of the input mutual intensity.

\section{Quadratic-Phase Systems}

In Ref. 10 it is discussed that systems involving several lenses separated by arbitrary distances of free space can also be analyzed by use of the fractional Fourier transform. More concretely, the kernel $h\left(x, x^{\prime}\right)$ characterizing these systems can also be written in terms of the fractional-Fourier-transform kernel.

We will consider the class of quadratic-phase systems that includes all systems composed of an arbitrary number of thin lenses separated by arbitrary sections of free space (in the Fresnel approximation). The kernel characterizing such a system is given by Refs. 10, 19, and 20:

$$
h\left(x, x^{\prime}\right)=C \exp \left[i \pi\left(\alpha x^{2}-2 \beta x x^{\prime}+\gamma x^{\prime 2}\right)\right],
$$

where $C$ is a complex constant and $\alpha, \beta$, and $\gamma$ are real constants. Such a kernel is sufficient to characterize the propagation of light from the input to the output or between any two planes of a quadratic-phase system. In Ref. 10 it is shown that the kernel given in Eq. (16) can be expressed in terms of the fractional Fourier transform, provided that we choose appropriate spherical reference surfaces. Thus, again using Eq. (5), we can easily express the mutual intensity between two planes in terms of the fractional Fourier transform.

The extension of these results to two-dimensional systems is straightforward, since the multidimensional fractional-Fourier-transform kernels are separable., Thus the relation between the input and output mutual intensities becomes a four-dimensional fractional Fourier transform.

\section{RELATION TO TRANSFORMATION OF THE WIGNER DISTRIBUTION}

Now we will discuss how the Wigner distribution associated with a partially coherent light distribution is transformed upon passage through an optical system. We will see that the result is perfectly analogous with the transformation law for the Wigner distribution associated with deterministic signals.

The Wigner distribution $W(x, \nu)$ of a deterministic field $U(\cdot)$ is defined as $^{5,21,22}$

$$
W(x, \nu)=\int U\left(x+x^{\prime} / 2\right) U^{*}\left(x-x^{\prime} / 2\right) \exp \left(-i 2 \pi x^{\prime} \nu\right) \mathrm{d} x^{\prime} .
$$

An important property of fractional Fourier transform is as follows: if $U_{\text {out }}$ is the fractional Fourier transform of $U_{\text {in }}$, i.e., if

$$
U_{\text {out }}(x)=k \int_{-\infty}^{\infty} B_{a}\left(\frac{x}{s_{2}}, \frac{x^{\prime}}{s_{1}}\right) U_{\text {in }}\left(x^{\prime}\right) \mathrm{d} x^{\prime},
$$

where $k$ is a complex constant and $s_{1}$ and $s_{2}$ are scale parameters, then

$$
\begin{aligned}
& W_{\text {out }}(x, \nu)=s_{1} s_{2}|k|^{2} W_{\text {in }}\left(\frac{s_{1}}{s_{2}} x \cos \phi-s_{1} s_{2} \nu \sin \phi,\right. \\
&\left.\frac{s_{2}}{s_{1}} \nu \cos \phi+\frac{1}{s_{1} s_{2}} x \sin \phi\right) .
\end{aligned}
$$

That is, performing the ath fractional Fourier transform corresponds to rotating the Wigner distribution by an angle $\phi=a(\pi / 2)$ in the clockwise direction..$^{6,5,9}$

We now derive the corresponding property for partially coherent light. In this case the Wigner distribution may be defined as

$$
\begin{aligned}
W(x, \nu)= & \int E\left[U\left(x+x^{\prime} / 2\right) U^{*}\left(x-x^{\prime} / 2\right)\right] \\
& \times \exp \left(-i 2 \pi x^{\prime} \nu\right) \mathrm{d} x^{\prime},
\end{aligned}
$$

which, using the definition of mutual intensity, becomes

$$
W(x, \nu)=\int J\left(x+x^{\prime} / 2, x-x^{\prime} / 2\right) \exp \left(-i 2 \pi x^{\prime} \nu\right) \mathrm{d} x^{\prime} .
$$

This is a Fourier-transform relation that can be inverted as

$$
J\left(x+x^{\prime} / 2, x-x^{\prime} / 2\right)=\int W\left(x, \nu^{\prime}\right) \exp \left(i 2 \pi x^{\prime} \nu^{\prime}\right) \mathrm{d} \nu^{\prime},
$$

which can be equivalently written as

$$
J\left(x_{1}, x_{2}\right)=\int W\left(\frac{x_{1}+x_{2}}{2}, \nu^{\prime}\right) \exp \left[i 2 \pi\left(x_{1}-x_{2}\right) \nu^{\prime}\right] \mathrm{d} \nu^{\prime} .
$$

In the previous sections we saw that with appropriate choice of input and output reference surfaces, $J_{\text {out }}\left(x_{1}, x_{2}\right)$ can be related to $J_{\text {in }}\left(x_{1}, x_{2}\right)$ as a two-dimensional fractional Fourier transform:

$$
\begin{aligned}
J_{\text {out }}\left(x_{1}, x_{2}\right)= & |k|^{2} \int_{-\infty}^{\infty} \int_{-\infty}^{\infty} B_{a}\left(\frac{x_{1}}{s_{2}}, \frac{x_{1}{ }^{\prime}}{s_{1}}\right) B_{-a}\left(\frac{x_{2}}{s_{2}}, \frac{x_{2}{ }^{\prime}}{s_{1}}\right) \\
& \times J_{\text {in }}\left(x_{1}{ }^{\prime}, x_{2}{ }^{\prime}\right) \mathrm{d} x_{1}{ }^{\prime} \mathrm{d} x_{2}{ }^{\prime} .
\end{aligned}
$$

Now, using Eqs. (24), (21), and (23), we find after some lengthy yet straightforward algebra that the relation between $W_{\text {out }}$ and $W_{\text {in }}$ is 


$$
\begin{array}{r}
W_{\text {out }}(x, \nu)=s_{1} s_{2}|k|^{2} W_{\text {in }}\left(\frac{s_{1}}{s_{2}} x \cos \phi-s_{1} s_{2} \nu \sin \phi,\right. \\
\left.\frac{s_{2}}{s_{1}} \nu \cos \phi+\frac{1}{s_{1} s_{2}} x \sin \phi\right),
\end{array}
$$

which is perfectly analogous to Eq. (19).

\section{CONCLUSION}

The mutual-intensity distribution is one of the most common ways of characterizing the spatial partial coherence of a wave field. In this paper we have shown how the propagation of mutual intensity through first-order optical systems (systems involving thin spherical lenses, quadratic graded-index media, and free-space propagation in the Fresnel approximation) can be expressed neatly in terms of the fractional Fourier transform. We have also seen that the Wigner distribution associated with these partially coherent fields rotates in the same manner as the Wigner distribution associated with a deterministic field, as one would intuitively expect. This extends the previous characterization of such optical systems in terms of the fractional transform in Ref. 10, to the case in which the partial coherence of light must be taken into account.

\section{REFERENCES}

1. A. C. McBride and F. H. Kerr, "On Namias's fractional Fourier transform," IMA J. Appl. Math. 39, 159-175 (1987).

2. H. M. Ozaktas and D. Mendlovic, "Fourier transforms of fractional order and their optical interpretation," Opt. Commun. 101, 163-169 (1993).

3. D. Mendlovic and H. M. Ozaktas, "Fractional Fourier transformations and their optical implementation. Part I," J. Opt. Soc. Am. A 10, 1875-1881 (1993).

4. H. M. Ozaktas and D. Mendlovic, "Fractional Fourier transformations and their optical implementation. Part II," J. Opt. Soc. Am. A 10, 2522-2531 (1993).

5. H. M. Ozaktas, B. Barshan, D. Mendlovic, and L. Onural, "Convolution, filtering, and multiplexing in fractional Fourier domains and their relation to chirp and wavelet transforms," J. Opt. Soc. Am. A 11,547-559 (1994).
6. A. W. Lohmann, "Image rotation, Wigner rotation, and the fractional Fourier transform," J. Opt. Soc. Am. A 10, 2181-2186 (1993).

7. A. Kutay, H. M. Ozaktas, L. Onural, and O. Arikan, "Optimal filtering in fractional Fourier domains," in Proceedings of the 1995 International Conference on Acoustics, Speech, and Signal Processing (IEEE, Piscataway, N.J., 1995), pp. 937-940.

8. A. W. Lohmann and B. H. Soffer, "Relationship between the Radon-Wigner and fractional Fourier transforms," J. Opt. Soc. Am. A 11, 1789-1801 (1994).

9. L. B. Almeida, "The fractional Fourier transform and timefrequency representations," IEEE Trans. Signal Process. 42, 3084-3091 (1994).

10. H. M. Ozaktas and D. Mendlovic, "Fractional Fourier optics," J. Opt. Soc. Am. A 12, 743-751 (1995).

11. P. Pellat-Finet, "Fresnel diffraction and the fractional-order Fourier transform," Opt. Lett. 19, 1388-1390 (1994).

12. P. Pellat-Finet and G. Bonnet, "Fractional-order Fourier transform and Fourier optics," Opt. Commun. 111,141-154 (1994).

13. L. M. Bernardo and O. D. D. Soares, "Fractional Fourier transforms and optical systems," Opt. Commun. 110, 517-522 (1994).

14. L. M. Bernardo and O. D. D. Soares, "Fractional Fourier transforms and imaging," J. Opt. Soc. Am. A 11, 2622-2626 (1994).

15. H. M. Ozaktas and D. Mendlovic, "The fractional Fourier transform as a tool for analyzing beam propagation and spherical mirror resonators," Opt. Lett. 19, 1678-1680 (1994).

16. B. E. A. Saleh and M. C. Teich, Fundamentals of Photonics (Wiley, New York, 1991).

17. J. W. Goodman, Statistical Optics (Wiley, New York, 1985).

18. M. Fatih Erden, Z. Zalevsky, D. Mendlovic, and H. M. Ozaktas, "Applications of the fractional Fourier transform to partially coherent systems," presented at the OSA Annual Meeting, Portland, OR, Sept. 10-15, 1995.

19. M. J. Bastiaans, "The Wigner distribution applied to optical signals and systems," Opt. Commun. 25, 26-30 (1978).

20. M. J. Bastiaans, "Wigner distribution function and its application to first-order optics," J. Opt. Soc. Am. A 69, 1710-1716 (1979).

21. F. Hlawatsch and G. F. Boudreaux-Bartels, "Linear and quadratic time-frequency signal representations," IEEE Signal Process. Magazine, 21-67 (April 1992).

22. H. O. Bartelt, K. H. Brenner, and A. W. Lohmann, "The Wigner distribution function and its optical production," Opt. Commun. 32, 32-38 (1980). 